\title{
Magnetospheric access for solar protons during the January 2005 SEP event
}

\author{
Vladimir V. Kalegaev ${ }^{1, *}$, Natalia A. Vlasova ${ }^{1}$, Ilya S. Nazarkov ${ }^{1}$, and Sophia A. Melkova ${ }^{2}$ \\ 1 Skobeltsyn Institute of Nuclear Physics of Lomonosov Moscow State University, Russia \\ 2 Physics Department of Lomonosov Moscow State University, Russia
}

Received 16 June 2017 / Accepted 19 September 2018

\begin{abstract}
The early phase of the extraordinary solar energetic particle 20 January, 2005 event having the highest peak flux of any SEP in the past 50 years of protons with energies $>100 \mathrm{MeV}$ is studied. Solar energetic particles $(>16 \mathrm{MeV})$ entry to the Earth's magnetosphere on January 20, 2005 under northward interplanetary magnetic field conditions is considered based on multi-satellite data analysis and magnetic field simulation. Solar wind parameters and interplanetary magnetic field data, as well as calculations in terms of the A2000 magnetospheric magnetic field model were used to specify conditions in the Earth's environment corresponding to solar proton event. It was shown that during the early phase of the event energetic particle penetration into the magnetosphere took place in the regions on the magnetopause where the magnetospheric and interplanetary magnetic field vectors are parallel. Complex analysis of the experimental data on particle fluxes in the interplanetary medium (data from ACE spacecraft) and on low-altitude (POES) and geosynchronous (GOES) orbits inside the Earth's magnetosphere show two regions on the magnetopause responsible for particle access to the magnetosphere: the near equatorial day-side region and open field lines window at the high-latitude magnetospheric boundary. Calculations in terms of A2000 magnetospheric magnetic field model and comparison with SuperDARN images support the link between high-latitude solar energetic particle precipitations and the region at the magnetopause where the magnetospheric field is coupled with northward IMF, allowing solar particles entrance into the magnetosphere and access to the northern polar cap.
\end{abstract}

Keywords: Magnetic field / Magnetosphere / Polar cap / Energetic particle / Reconnection

\section{Introduction}

Measuring the variations of the solar energetic particle (SEP) fluxes inside the magnetosphere is an excellent tool for a comprehensive study not only of the interplanetary medium state, but also of the Earth's magnetospheric structure and dynamics (see, e.g., Darchieva et al., 1983; Flückiger et al., 1990; Blake et al., 2001; Tverskaya, 2011). Variations of the large-scale magnetospheric currents in accordance with both, interplanetary magnetic field and solar wind changes, affect the particle transport and distribution inside the magnetosphere (Harnett, 2010; Rodriguez, 2012). The most significant changes in the magnetosphere take place during magnetic storms when SEP fluxes are registered at latitudes lower than under quiet conditions (Flückiger et al., 1990; Kudela et al., 2008). Substorm activity and solar wind pressure pulses can also enhance proton

\footnotetext{
*Corresponding author: klg@decl.sinp.msu.ru
}

access to low $L$-shells due to magnetospheric magnetic field reconfiguration (Paulikas \& Blake, 1969; Rodriguez, 2012). Due to large Larmor radius solar particles can move nonadiabatically in the regions where the field lines are highly curved. In that way particles can shift from the open to closed field lines and become trapped by geomagnetic field (Richard et al., 2009).

The most famous basis of present solar-terrestrial physics is the conception of the open magnetosphere (Dungey, 1961). It is assumed that magnetospheric field lines can reconnect with interplanetary magnetic field (IMF) lines. Such coupling can provide the solar wind mass, energy and momentum transfer through the Earth's magnetopause. This is also the way for charged particle entry into the magnetosphere (see, e.g., Farrugia et al., 1993; Merkin \& Goodrich, 2007 and reference therein). Paulikas (1974) reported that measurements of solar proton fluxes in the high polar latitude region give strong evidence in favor of the direct connection between IMF and 
magnetospheric magnetic field. Two regions on the magnetopause give access for solar protons inside the magnetosphere. The first one is located on the near-equatorial dayside magnetopause while the second one corresponds to the region on the magnetopause intersected by open magnetic field lines. These two "windows" can provide energetic particle access to low and high latitudes respectively.

IMF $B_{z}$-component controls the energy input rate and responsible for electrodynamic processes taking place in the Earth's magnetosphere (see, e.g., Lemaire et al., 2005). Depending on sign of $B_{z}$ ("northward" or "southward" IMF) the different mechanisms of solar wind - magnetosphere coupling will be realized. In the same way, different magnetospheric magnetic field structures determining the charged particle motion and distribution inside the magnetosphere will take place. In the framework of MHD model of solar wind magnetosphere interaction Richard et al. (2002) demonstrated that IMF plays the crucial role in SEPs entry into the magnetosphere. The most complicated geomagnetic field structure arises under northward IMF (Cowley, 1973; Belenkaya, 2004) when charged particle penetration into the Earth's magnetosphere is impeded.

A classical theory of charged particle motion in the dipole magnetic field has been developed by C. Störmer (1955). One can use it to describe roughly the main features of solar energetic particle penetration into the Earth's magnetosphere. However, the magnetic field in the Earth's environment is not a dipolar one and changes in response to solar wind conditions. Direct measurements show that energetic protons can penetrate at more low latitudes than predicted by Störmer's theory (Paulikas \& Blake, 1969). Real geomagnetic field and its dynamics produce some corrections to the particle motion. Magnetospheric current systems as well as interplanetary magnetic field have an influence on the size and location of the regions in the magnetosphere that can be accessed by solar particles (Richard et al., 2002).

Direct entry along the open magnetic field lines is the basic mechanism describing penetration of the solar protons into the magnetosphere (Paulikas, 1974). However, the high-energy particles can also come into the magnetosphere across magnetic field lines by slow diffusion-like process (see review Scholer, 1975 and references therein). Two ways for solar energetic particles access to the Earth's magnetosphere were suggested in Tverskoy et al. (1973): penetration into polar caps along IMF lines reconnected with magnetospheric magnetic field (from high-latitude magnetopause), and penetration into the auroral and subauroral regions due to diffusion mechanism (from the distant tail). Results of particle tracking (Richard et al., 2002) show that protons with energies less than $10 \mathrm{MeV}$ can access the magnetosphere along open field lines, while the more energetic particles can directly penetrate magnetosphere on the dayside magnetopause due to large Larmor radii. Diffusive entry of solar flare particles into geomagnetic tail has been studied in Michel \& Dessler (1970). Diffusion results from the magnetic field fluctuations. Another mechanism of prompt trapping of SEPs was presented in Kress et al. (2005) for the storm-time periods. Solar energetic particles can be impulsively injected into the inner $L$-shells by sudden compression of the magnetosphere. The results indicate that an enhancement in solar wind dynamic pressure for this event plays a role in the observed injection of ions to low $L$-values (Tverskoy, 1969; Pavlov et al., 1993; Hudson et al., 2004).

Results of experimental study of solar energetic particle penetration events display great variety of observed dynamical behavior of particle fluxes (see, e.g., a review Pereyaslova (1982) and references therein). In particular, solar proton fluxes can be non-uniform in the magnetospheric polar region. In such cases, the profiles of SEP particle fluxes in the polar cap, auroral oval and cusp areas can have different structures that allow to distinguish these regions (Pereyaslova et al., 1978). Observed structures can be reproduced by simulations in the framework of magnetospheric magnetic field models that can help to reconstruct the particle motion taking into account the diffusion as well as direct penetration along reconnected field lines (see, e.g., Morfill \& Quenby, 1971; Blake et al., 2001; Richard et al., 2009; Desorgher et al., 2009).

The modern magnetospheric models allow to describe magnetic field structure for actual interplanetary medium conditions (see, e.g., Alexeev et al., 2001; Tsyganenko, 2002; Tsyganenko \& Sitnov, 2005). However, to represent correct real solar wind - magnetosphere interaction we need also a model of the magnetosheath's magnetic field that can help to reproduce interplanetary and magnetospheric magnetic fields coupling. The A2000 magnetospheric model (Alexeev et al., 2001) together with coupled model of magnetosheath magnetic field (Alexeev \& Kalegaev, 1995) allow to calculate the global magnetic field structure taking into account Dungey-type diffusional reconnection at the magnetopause. The model of magnetosheath magnetic field (Alexeev \& Kalegaev, 1995) takes into account the reconnection rate ("reconnection efficiency") depending on solar wind plasma density and conductivity near the magnetopause.

The goal of this paper is to explain specific features of solar particle penetration observed on January 20, 2005. This SEP event was the unusual one in solar cycle 23: the associated $\mathrm{X}$-ray flare was the fifth strongest and it has the highest CME speed; the highest peak fluxes of any SEP in the past 50 years were registered for protons, helium and heavy ion populations; event was observed on the ground as GLE \#15, most intensive in solar cycle 23 .

Multi-satellite and on-ground measurements give opportunity to clarify mechanism of SEP penetration into the Earth's magnetosphere in more detail. We will study early stage of SEP event on the base of experimental data from NOAA/ POES, GOES and ACE satellites, together with the magnetospheric model A2000 calculations. NOAA/POES-17 spacecraft measured the time profile of solar proton fluxes just during onset of the SEP event. Magnetospheric magnetic field configuration during the event reveals itself in the specific "channeled" structure of the SEP flux time profiles in the Northern hemisphere. Magnetic field calculations gave opportunity to precisely distinguish the different patterns in SEP profiles and determine the corresponding areas of SEP penetration at the magnetopause. Polar cap reconstruction by SuperDARN as well as magnetic field structure calculated by the A2000 magnetospheric model demonstrates and explains the ways of the SEP access into the magnetosphere under northward IMF. 
Table 1. Spacecrafts, their orbits and the energies of measured proton fluxes.

\begin{tabular}{llr}
\hline Spacecrafts & Orbit & Proton energy, MeV \\
\hline ACE & Libration point $L 1-$ & $>10$ \\
& 1.5 million km from the Earth to the Sun & $>30$ \\
POES-15 (LTAN 17:06:00) & Polar Sun-synchronous; & $>16$ \\
POES-16 (LTAN 16:35:00) & Altitude: $\sim 800 \mathrm{~km}$ & $>36$ \\
POES-17 (LTAN 22:03:00) & Inclination Angle: $999^{\circ}$ & $>70$ \\
(LTAN is Local Time of the Ascending Node) & Period: $\sim 100$ min & Geosynchronous; \\
GOES-10, 11, 12 & Altitude: $\sim 36000 \mathrm{~km}$, & $>10$ \\
& Inclination: $\sim 0^{\circ}$, & $>30$ \\
& Longitude: $135^{\circ} \mathrm{W}, 135^{\circ} \mathrm{W}, 60^{\circ} \mathrm{W}$ (respectively) & $>100$ \\
\hline
\end{tabular}

\section{Experimental Data}

Presented results were obtained on the base of experimental data of solar particle fluxes measured by ACE, NOAA/POES and GOES spacecrafts (see Table 1).

Data on the interplanetary medium parameters were obtained from ACE spacecraft. Geomagnetic indices variations: Dst, $A S Y-H, S Y M-H$ and $A L$ (World Data Center C2 for Geomagnetism, Kyoto (http://wdc.kugi.kyoto-u.ac.jp/)), were considered to estimate the level of geomagnetic activity.

\section{Results}

SEP event on January 20, 2005 was one of the events related to prolonged period of solar activity 15-20 January, 2005. Figure 1 shows solar proton fluxes registered by ACE and GOES-10 spacecraft during this period. Dashed lines bound the time interval between 19 and 20 January 2005. We will study the SEP event with the particle flux maximum that was registered approximately at 06:55 UT on January 20, 2005. The source of this SEP event was the solar flare: 20d06h36m, $X 7.1 / 2 B, N 12 W 58, A R 10720$ (Logachev et al., 2016). Solar proton flux outside the magnetosphere was highly anisotropic during onset of SEP event (Bütikofer et al., 2008). One can see from the Figure 1 an important detail: when SEP event started the solar particle fluxes of previous events (taking place on 17 January and earlier) still existed in the interplanetary medium as well as in the magnetosphere. Particle fluxes related to the event on 20 January (the "fresh" particle fluxes) began to increase on the background of relatively high level of particle fluxes originated from the earlier SEP events (the "old" particle fluxes).

Interplanetary and geomagnetic conditions during this SEP event are presented in Figure 2. Magnetospheric state at the beginning of the SEP event was quiet $(A L, A S Y / H$ and $S Y M / H$ were near zero) and magnetopause stand-off distance (calculated by Shue et al., 1998 model) was about $13 R_{E}$ due to very low solar wind pressure (about of $0.5 \mathrm{nPa}$ ). It is very essential to take into account that IMF was practically northward ( $B_{z}>0$, while $B_{x}$ and $B_{y}$ were near to zero) before and at the onset of SEP event ( 16:55 UT on January 20, 2005).

Experimental data obtained from three POES satellites give the excellent opportunity to study SEP penetration features (Fig. 3) during this event. SEP fluxes onboard POES can be measured when the satellite is moving over the polar regions. One can see that particle fluxes were enhanced already prior 06:40 UT when POES satellites detected the particles originated from earlier SEP events - the "old" particle fluxes. After the solar eruption occurred at 06:36 UT, energetic particle fluxes in the Earth's environment drastically changed. As the solar flare occurred at the west solar longitudes (W58) the arrival of SEP on Earth orbit was very fast. Time profiles of SEP fluxes measured onboard ACE and GOES spacecrafts help us to define the beginning of the particle flux increase phase, approximately at 06:55 UT on 20 January, 2005. Fortunately, POES-17 was over the northern polar cap just at arrival time and observed SEP event development at low altitudes during the single polar region crossing. One can see the unusual and specific time profile of SEP fluxes in polar region: "channeled" structure which has four maxima and three minima between them. Particle flux profiles measured in polar cap onboard POES-16 and POES-15 after the increase phase have also a complicated but usually observed structure: two maxima and one minimum in the polar cap center.

The "channeled" structure of particle fluxes measured onboard POES-17 during 06:40-07:20 UT is presented in Figure $4 \mathrm{a}$ in more detail. Time profiles of particle fluxes measured onboard ACE $(>10$ and $>30 \mathrm{MeV})$ and GOES-10 $(>50 \mathrm{MeV})$ are also shown by dots, asterisks and triangles, respectively. Maximum fluxes measured by POES-17 correspond well to the fluxes measured by GOES-10 and apparently related to SEP event on 20 January. Figure $4 \mathrm{~b}$ shows 15-44 MeV and 40-80 MeV SEP differential fluxes measured by GOES-10, GOES-11 and GOES-12. One can see that oppositely directed detectors onboard GOES-10 (eastward) and GOES-11 (westward) represent the same fluxes during SEP event onset. At UT 06:53 (first maximum) POES 17 observed entry of SEP in the inner magnetosphere and integral flux of $140 \mathrm{MeV}$ particles was about $100\left(\mathrm{~cm}^{2} \cdot \mathrm{s} \cdot \mathrm{sr}\right)^{-1}$. Figure $4 \mathrm{~b}$ shows that at this moment GOES 10 and GOES 11 registered background fluxes of particles in the both 15-44 MeV and 40-80 MeV channels. Apparently, at UT 06:53 15-44 MeV and 40-80 MeV SEP did not penetrate inside the inner magnetosphere unlike the more energetic SEP observed by POES. Figure $4 \mathrm{~b}$ shows that substantial fluxes of low energy SEPs appeared in the inner magnetosphere only after UT 07:00.

Comparison of the fluxes measured at geostationary and polar orbits shows the existence of the different access pathways for the particles detected at high and low latitudes. The channeled fluxes structure measured by polar satellite reflects 


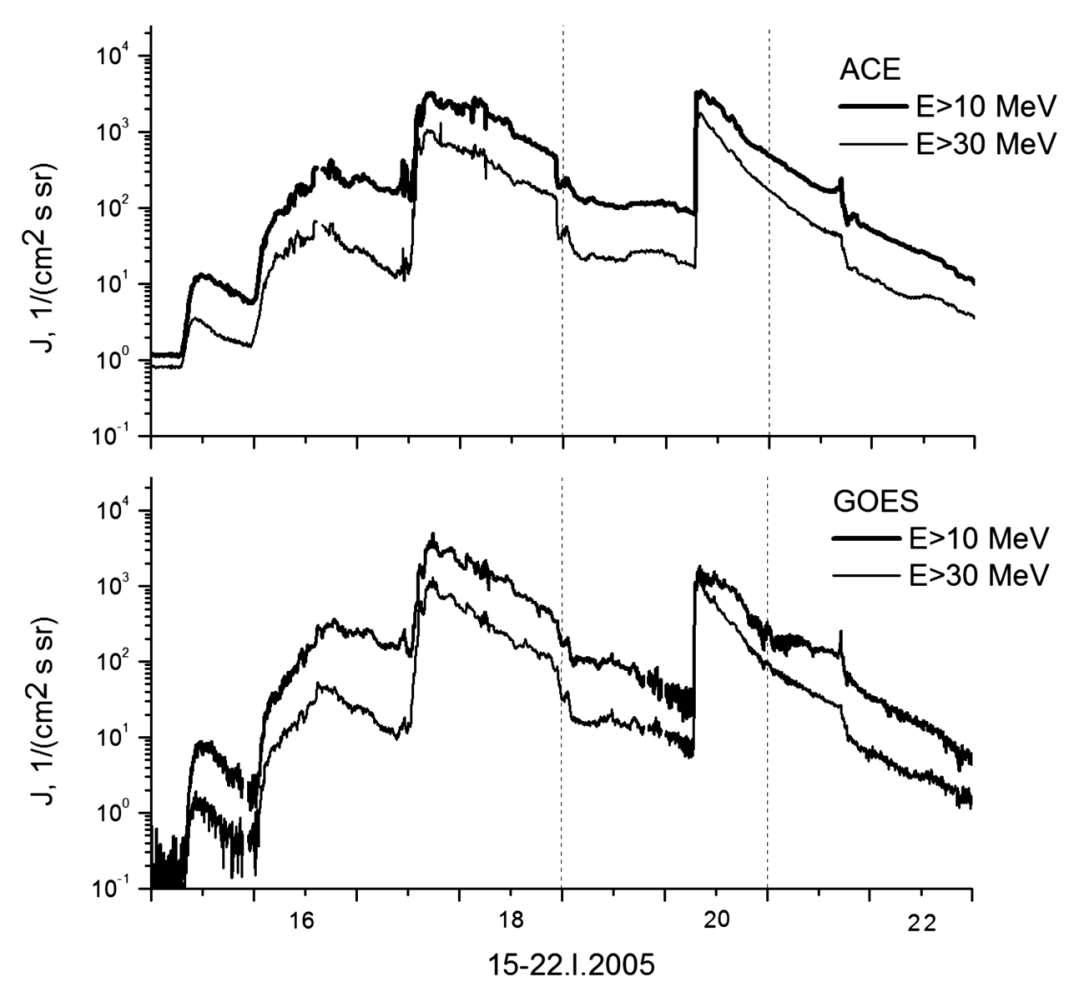

Fig. 1. Time profiles of SEP fluxes measured by ACE and GOES-10 on January 15-22, 2005. Dashed lines bound the time interval under consideration.

the magnetic field topology that arises near 07:00 UT and influenced particle penetration to the high latitudes. Correspondence with GOES measurements confirms that this structure is not the result of temporal variations of the particle fluxes, east-west SEP anisotropy, flux saturation or "dead time" effect.

So, Figures 3 and 4a show that POES-17 measured fluxes of two different populations of the energetic particles during the crossing of the polar region. The temporal development of maximum particle fluxes reproduces well the external fluxes variations measured by ACE and GOES. To understand better the origin of the particles measured in the region of minima of the fluxes, $L$-profiles of particle fluxes of protons with energies more than $16 \mathrm{MeV}$ and $36 \mathrm{MeV}$ measured by POES-17 during inbound crossing of polar region ( 06:40-07:00 UT) are presented (see Fig. 4c blue and red thick lines respectively). One can see also profiles of the "old" fluxes of particles with the same energies measured by POES-17 during previous inbound crossing of the northern polar cap during 04:50-05:10 UT (see thin lines in Fig. 4c). Particle fluxes measured in the first minima of the "channeled" structure coincide exactly with the "old" particles fluxes for both energetic channels.

One can see that during the onset of SEP event "fresh" particle accessed the narrow regions near some specific $L$-shells (where the maximum fluxes have been detected) by the most direct and therefore, the quickest pathways. Obviously, such distribution corresponds to the existing magnetospheric magnetic field structure. One can see the same fluxes in different energetic channels: so the particle spectrum in maxima is almost flat. We believe that in maxima all the POES-17 particle detector channels registered mostly the fluxes of the "fresh" energetic protons with energies about of $100 \mathrm{MeV}$ and higher. On the other hand, particle fluxes in the minima have the falling profile of the spectra. The different spectral characteristics in maxima and minima support our conclusion on different origin of the particles measured in polar region at different $L$-shells. One can see however, that in the next minima the particle fluxes become higher. During the next northern polar cap crossing POES-17 measured the particle fluxes distribution usually detected during proton events. It means that distribution of the fluxes across the polar region measured at 06:50-07:10 UT is unique and related apparently to the specific solar wind conditions and specific characteristics of particle fluxes at the onset of SEP event.

Positions of the observed maxima and minima of particle fluxes are very important for explanation of the "channeled" structure. Table 2 contains the geographic and magnetic latitudes, the $L$-parameter, MLT and flux amplitudes measured by satellite in the time moment noted in Figure 4a by numbers of 1-7. Consequent enhancement of fluxes in 1, 3, 5, 7 points (maxima) registered during POES-17 polar fly-by on 6:40-7:20 UT reflects exactly the SEP event development measured by GOES satellites. One can see that point No1 (maximum) observed at the dusk side of the magnetosphere is located approximately at the same low magnetic $L$-shell as the dayside point No7 (maximum). Two points - No3 and No5 (maxima) are located also on the similar $L$-shells (similar invariant latitudes) but in the polar region. It means that particles near the points No1 and No7 belong to the same $L$-shells, as well as particles near the points No3 and No5. They have also the same invariant latitudes, $71^{\circ}$ and $82^{\circ}$ respectively. 


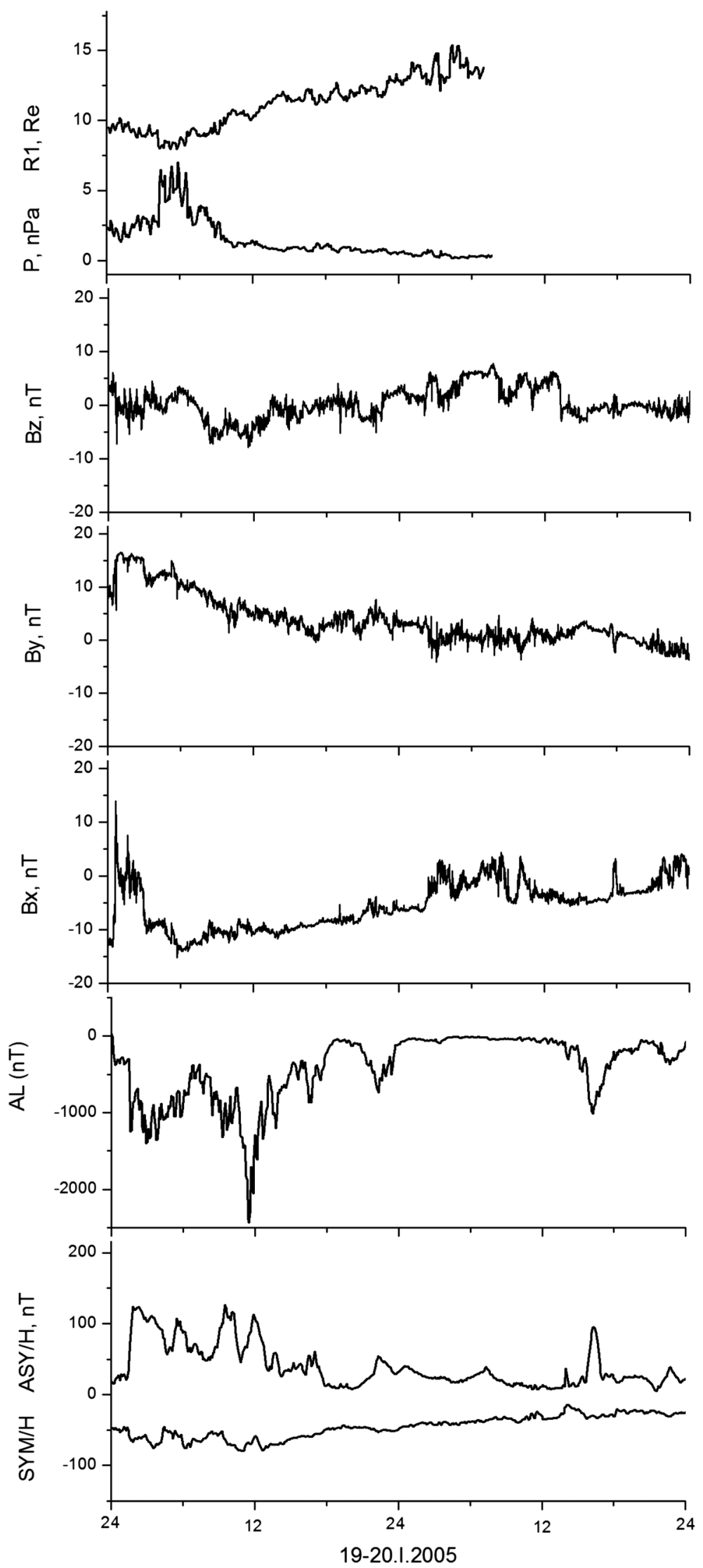

Fig. 2. Time profiles of interplanetary medium and magnetospheric key parameters: solar wind pressure $(P)$ and distance to the subsolar point $\left(R_{1}\right)$, IMF components $\left(B_{z}, B_{y}, B_{x}\right)$, and geomagnetic indices: $A L, A S Y / H$ and $S Y M / H$ on January 19-20, 2005. 


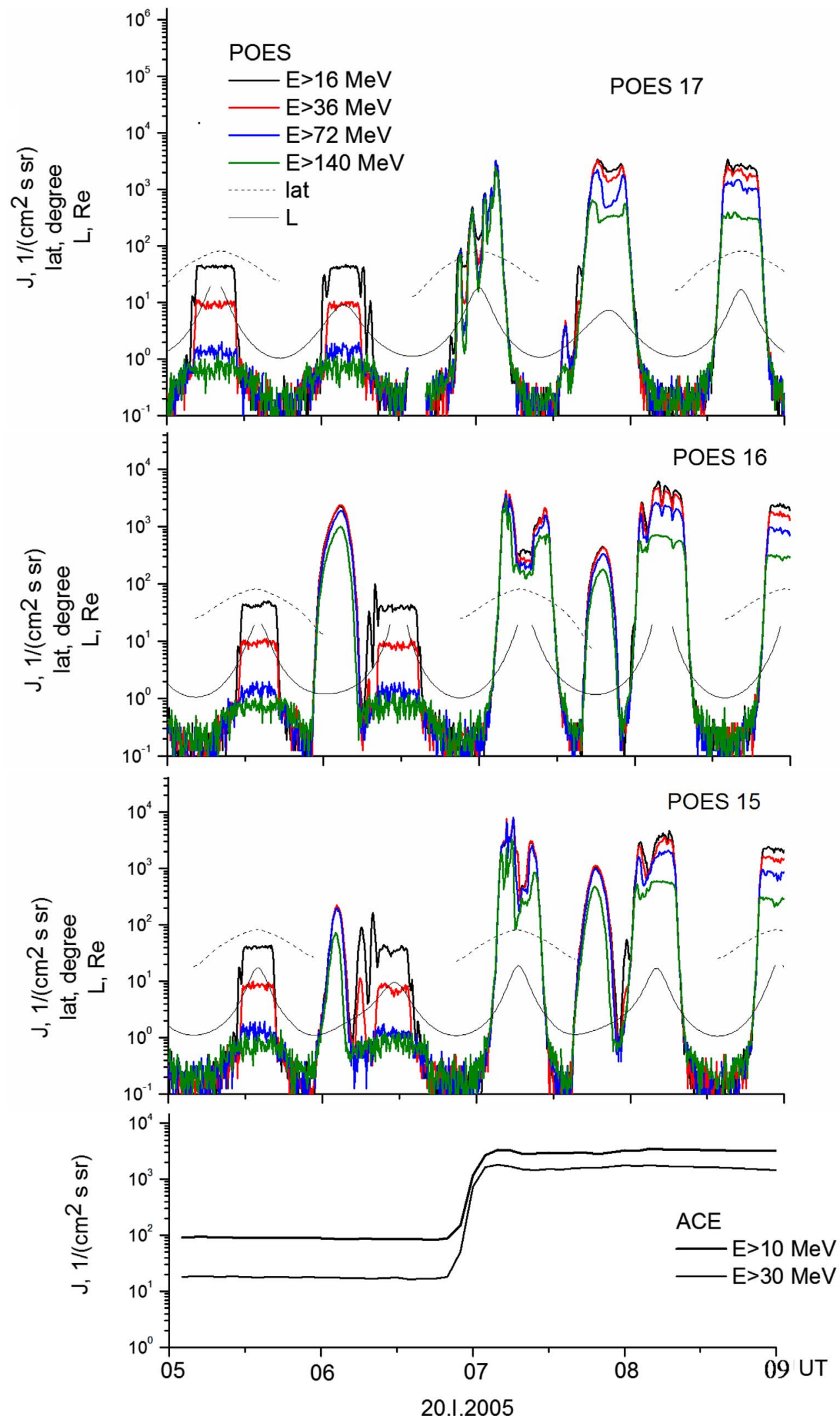

Fig. 3. Time profiles of SEP fluxes measured onboard POES and ACE spacecrafts at 05:00-08:00 UT on January 20, 2005. 


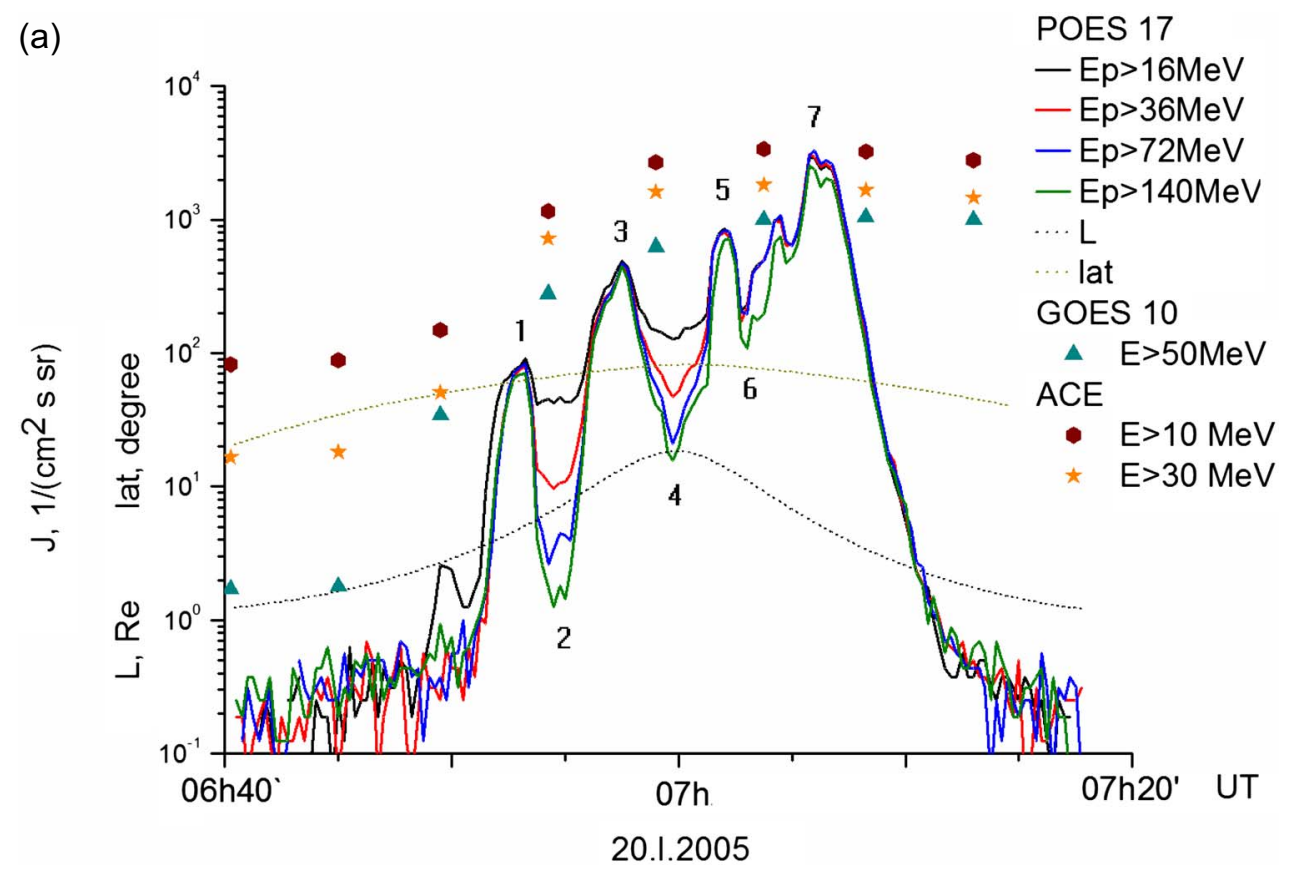

(b)

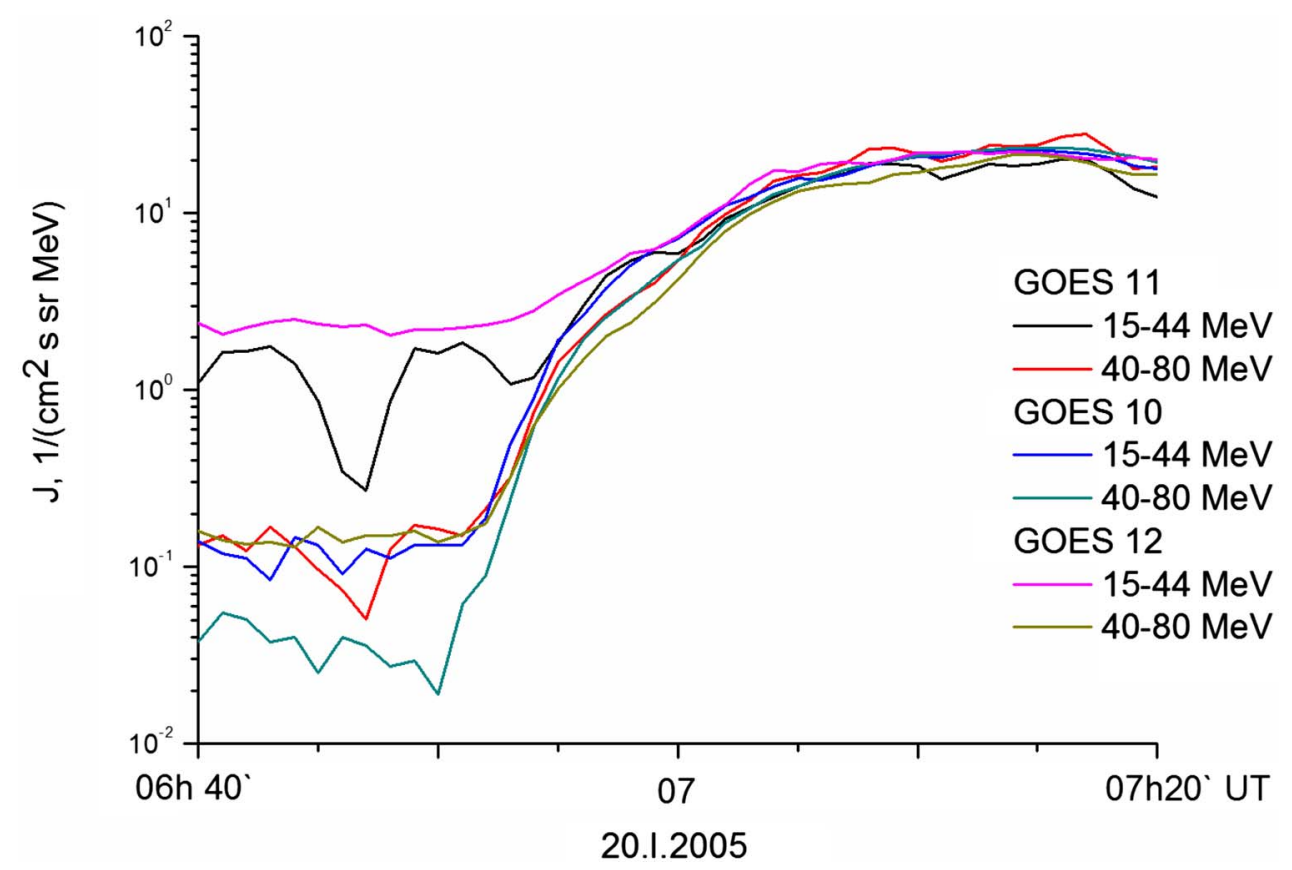

Fig. 4. (a) Time profiles of SEP fluxes measured onboard POES-17, ACE and GOES-10 spacecraft and of parameters of POES-17 orbit: MLT, the geographic latitude of foot-of-field-line and the $L$-parameter. (b) Time profiles of SEP fluxes measured onboard GOES-10, GOES-11 and GOES-12 spacecraft. (c) L-profiles of SEP fluxes measured onboard POES-17 (see text).

So, analysis of experimental data measured by NOAA/ POES 17 satellite during SEP event onset shows that there exist two separated populations of solar particles in the Earth's magnetosphere coming from solar flare that occurred at 06:36 UT on 20 January 2005 . First one is located at low $L$ shells while the second one belongs to the open magnetic field region.

\section{Discussion}

The main properties of SEP fluxes coming to the Earth's environment are influenced by solar flare intensity and location on the Sun as well as by physical conditions in the interplanetary medium. These factors determine the propagation time 


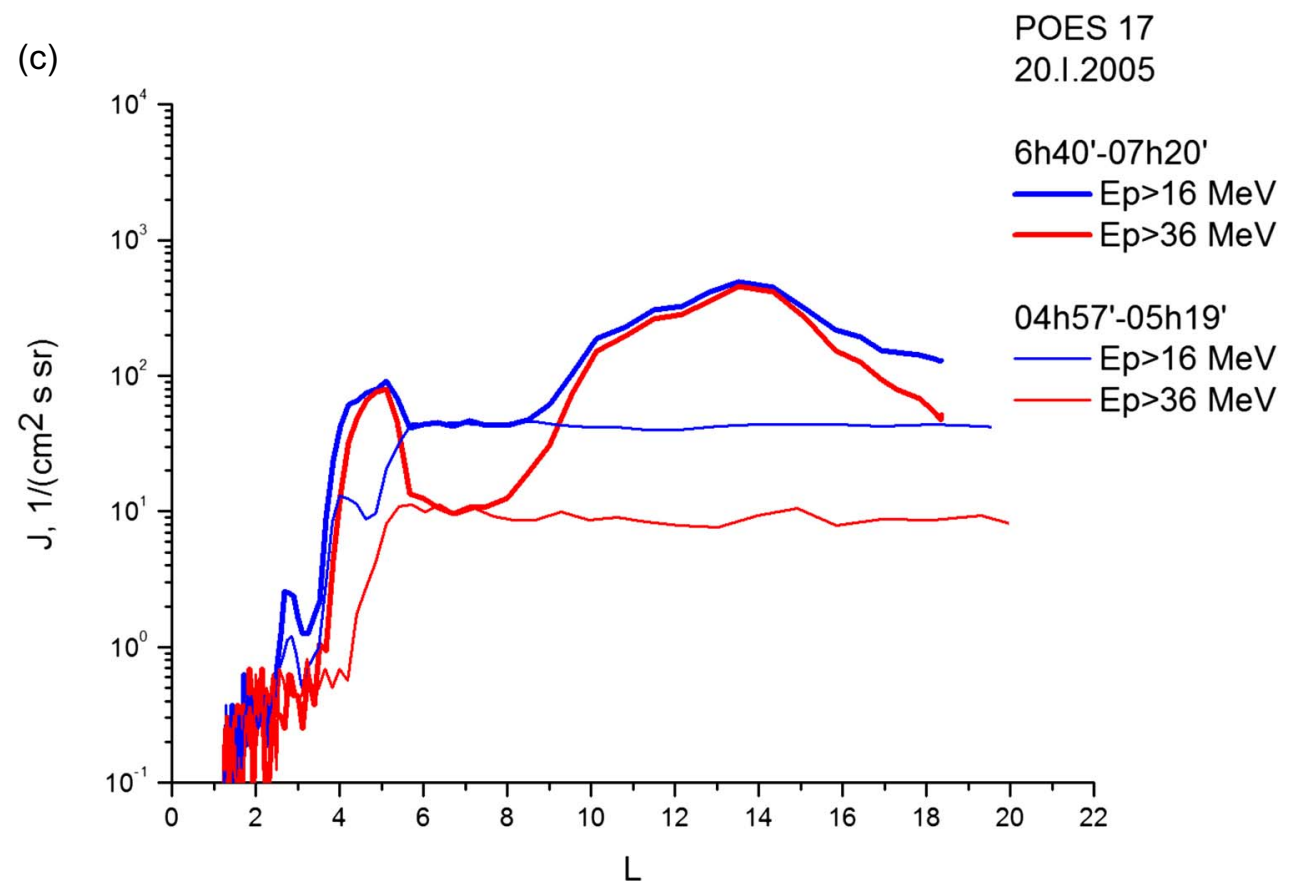

Fig. 4. Continued.

Table 2. Parameters of the "channeled" structure.

\begin{tabular}{ccccccccc}
\hline No & & UT & MLat, degree & Lat, degree & $L, R_{E}$ & MLT, hours & $J,\left(\mathrm{~cm}^{2} \mathrm{~s} \mathrm{sr}\right)^{-1}\left(E_{p}>72 \mathrm{MeV}\right)$ & $J,\left(\mathrm{~cm}^{2} \mathrm{~s} \mathrm{sr}\right)^{-1}\left(E_{p}>140 \mathrm{MeV}\right)$ \\
\hline 1 & Max & $06: 53: 56$ & 64.05 & 62.45 & 5.120 & 21.1 & $8.288 \times 10^{1}$ & $7.088 \times 10^{1}$ \\
2 & Min & $06: 55: 00$ & 66.62 & 65.83 & 6.340 & 20.7 & $2.625 \times 10^{0}$ & $1.750 \times 10^{0}$ \\
3 & Max & $06: 58: 28$ & 72.58 & 76.24 & 13.51 & 18.6 & $4.699 \times 10^{2}$ & $4.459 \times 10^{2}$ \\
4 & Min & $07: 00: 52$ & 72.94 & 81.24 & 18.34 & 16.0 & $2.112 \times 10^{1}$ & $1.569 \times 10^{1}$ \\
5 & Max & $07: 03: 16$ & 69.86 & 80.18 & 14.00 & 14.0 & $8.359 \times 10^{2}$ & $6.959 \times 10^{2}$ \\
6 & Min & $07: 04: 20$ & 67.71 & 77.86 & 11.18 & 13.4 & $1.974 \times 10^{2}$ & $1.076 \times 10^{2}$ \\
7 & Max & $07: 07: 32$ & 59.78 & 68.76 & 5.370 & 12.4 & $3.264 \times 10^{3}$ & $2.372 \times 10^{3}$ \\
\hline
\end{tabular}

from the Sun to the Earth's orbit as well as main mechanisms of particle entry into the magnetosphere. The solar flare on January 20, 2005 has occurred on the western solar longitudes and, as a result, solar protons arrived to the Earth quickly and the particle flux time profile had a sharp increase. Interplanetary medium conditions were quiet (Fig. 2) so, flux anisotropy could achieve $\sim 100 \%$ at the beginning of the event (Miroshnichenko \& Petrov, 1985; Bütikofer et al., 2008).

Solar particles access into the near-Earth's environment depends on physical conditions in solar wind and in the magnetosphere. Quiet interplanetary conditions during the beginning of 20 January 2005 provided prolonged stable state of the magnetosphere during at least $6 \mathrm{~h}$ prior the solar flare. Low solar wind pressure and IMF with dominant $B_{z}>0$ set magnetospheric magnetic field configuration typical for northward IMF. It is known that under northward IMF magnetospheric magnetic field contains two neutral points near the magnetopause above the cusps (Cowley, 1973; Alexeev et al., 2007). Figure 5 shows magnetic field structure (Fig. 3 from Alexeev et al., 2007, modified Fig. 1e from Cowley, 1983) that arises under northward IMF. There are two regions on the magnetopause above the cusps where IMF is antiparallel to the magnetospheric magnetic field and can reconnect with it. Reconnected magnetic field lines go from the southern part of the magnetopause to the northern polar cap and vise-versa (see, e.g., Belenkaya, 2004). Solar wind electric field is transported along the open magnetic field lines to the ionospheric level. Unlike the case of southward IMF, ionospheric convection patterns during the northward IMF contain the parts with sunward-directed flow at high latitudes (Cowley, 1973; Potemra et al., 1984; Alexeev et al., 2007).

Magnetic field lines are the pathways for the charged particles. Open structure of the magnetospheric magnetic field formed due to reconnection provides the particle access from interplanetary medium to the inner magnetosphere. Quasidynamical quantitative magnetospheric models (Alexeev et al., 2001; Tsyganenko, 2002; Tsyganenko \& Sitnov 2005) give possibility to understand better mechanisms of the particle transport through the magnetopause.

A2000 model of the magnetosphere (Alexeev et al., 2001) allows to reproduce variations of the magnetospheric magnetic field under the different conditions in the solar wind. 


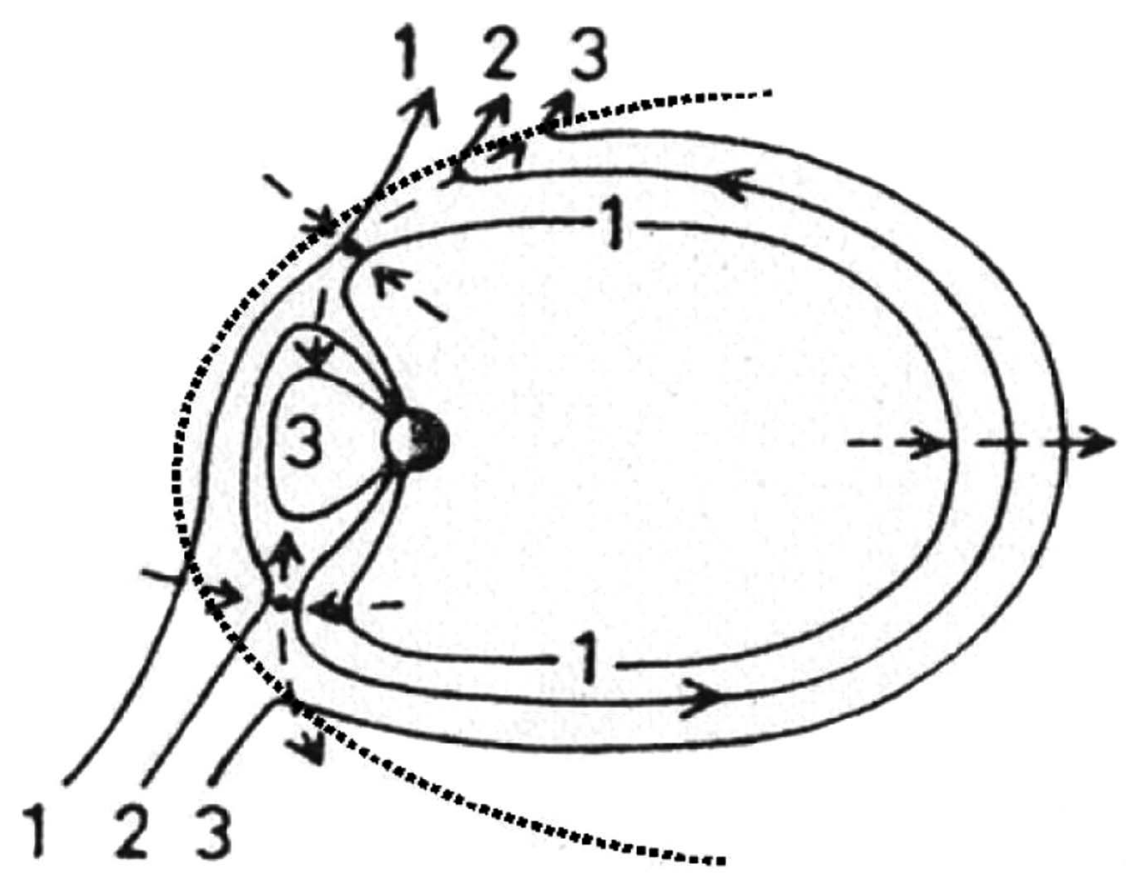

Fig. 5. Magnetic reconnection under northward IMF (by Alexeev et al., 2007).

The A2000 paraboloid model represents the magnetic field in near-Earth's space as a superposition of contributions from large-scale current systems, based on analytical solutions of the Laplace equations for each large scale current system with the boundary condition $B_{n}=0$ on the magnetopause, which is approximated by a paraboloid of revolution (Alexeev et al., 2001). In the paraboloid A2000 model the magnetic field reads $B=B_{\mathrm{m}}+B_{\mathrm{IGRF}}$. Here $B_{\mathrm{IGRF}}$ is the internal magnetic field produced by inner-Earth's currents, while $B_{\mathrm{m}}$, magnetic field of magnetospheric sources (currents on the magnetopause $\left(B_{\mathrm{cf}}\right)$, magnetotail currents $\left(B_{\mathrm{t}}\right)$, ring current $\left(B_{\mathrm{r}}\right)$, and field-aligned currents $\left.\left(B_{\mathrm{fac}}\right)\right)$, is determined as a sum:

$$
\begin{aligned}
\boldsymbol{B}_{\boldsymbol{m}}= & \boldsymbol{B}_{\mathrm{cf}}\left(\boldsymbol{\psi}, \boldsymbol{R}_{\mathbf{1}}\right)+\boldsymbol{B}_{\mathrm{t}}\left(\boldsymbol{\psi}, \boldsymbol{R}_{\mathbf{1}}, \boldsymbol{R}_{\mathbf{2}}, \boldsymbol{\Phi}_{\infty}\right)+\boldsymbol{B}_{\mathrm{r}}\left(\boldsymbol{\psi}, \boldsymbol{b}_{\boldsymbol{r}}\right) \\
& +\boldsymbol{B}_{\mathrm{fac}}\left(\boldsymbol{I}_{\|}\right) .
\end{aligned}
$$

The model input parameters are: tilt-angle $\psi$ of geomagnetic dipole, distance $R_{1}$ to the subsolar point on the magnetopause, distance $R_{2}$ to the inner edge of the magnetotail current sheet, magnetic flux $\Phi_{\infty}$ through the magnetotail lobes, magnetic field $b_{r}$ at the Earth's center produced by the ring current, and maximum intensity $I_{\|}$of Region 1 field-aligned currents. They characterize the intensitis of magnetospheric current systems and their positions in space. Each magnetic field source depends on its own set of parameters, which determines its unique dynamics under the variable solar wind conditions and geomagnetic activity. The model parameters can be determined from data of measurements of solar wind plasma and IMF, and from geomagnetic indices.

Figure 6 shows magnetic field line structure in the noonmidnight plane under interplanetary conditions taken at 07:00 UT on January 20, 2005 that was calculated by the A2000 magnetospheric model taking into account northward IMF penetration through the magnetopause based on Alexeev \& Kalegaev (1995) model. Red and green colors mark open and closed field lines, respectively. Blue lines show broken IMF lines that cross the magnetosphere. One can see that the region on the magnetopause crossed by open magnetic field lines is relatively small under northward IMF. In Sun-Earth's direction its length is about $50 R_{E}$. Transverse size of this region is also about $40 R_{E}$. Energetic particles that enter into the magnetosphere through this region on the Southern magnetopause can reach the Northern polar cap and contribute to fluxes measured by low Earth's orbit satellites.

We suppose that solar particle flux anisotropy during onset of the SEP event as well as specific magnetospheric structure formed during prolonged quiet conditions under northward IMF were the main factors responsible for above-mentioned penetration features measured by POES-17 after 06:40 UT on January 20, 2005. Previous studies (Tverskoy, 1973; Paulikas, 1974; Richard et al., 2002, 2009; Rodriguez, 2012) show that solar particle penetration into the Earth's high-latitude magnetosphere during SEP event can occur along the open field lines and also by diffusion-like mechanisms. Richard et al. (2002) reported that high-energy particles can directly penetrate magnetosphere on the dayside magnetopause due to large Larmor radii, while the low energy SEPs $(<10 \mathrm{MeV})$ can access magnetosphere moving along the open field lines. Effectiveness of such entry depends strongly on IMF direction (northward IMF is not "comfortable" for particle entry) and on the area of entry window on the magnetopause, that is relatively small for northward IMF (Paulikas, 1974). Figure 6 demonstrates that open magnetic field lines that connect entry window on the magnetopause with high latitude ionosphere were long enough at onset of SEP event on 20 January 2005. Such magnetic configuration prevents the fast entry of low energy SEPs into the high latitude magnetosphere. 


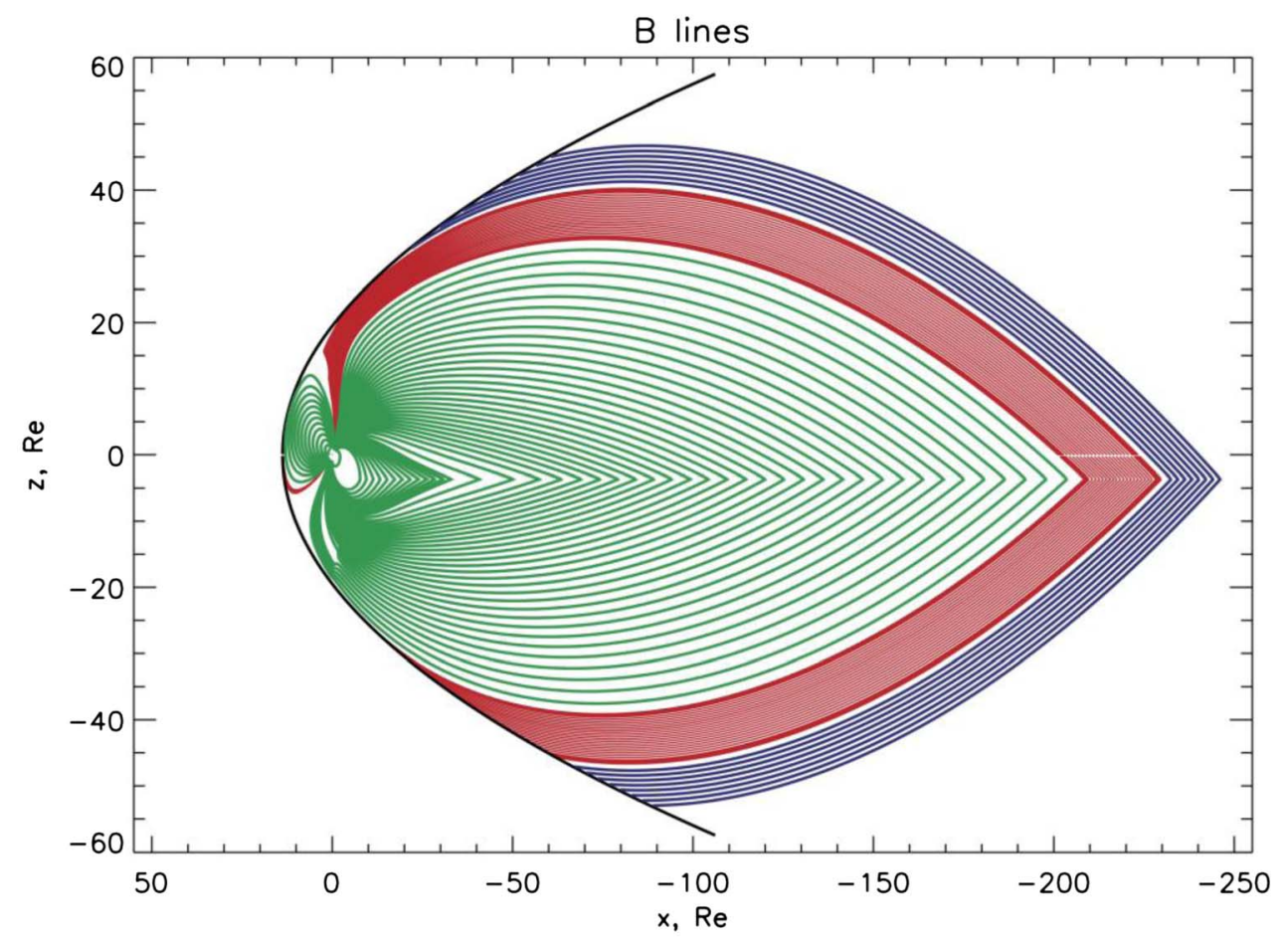

Fig. 6. A projection of magnetic field lines of the Earth's magnetosphere on the $X Z$-plane calculated by the A2000 magnetospheric magnetic field model for 06:00-07:00 UT on January 20, 2005 (see text).

For the anisotropic, bidirectional fluxes registered during the onset of the SEP event (Bütikofer et al., 2008) most of the particle momentum was directed along the field line, with only a small portion of the momentum directed toward the magnetosphere, making it difficult for low energy particles to penetrate. For particles with high enough energy, the penetration was most effective in the region on the magnetopause where the IMF was parallel to the adjacent magnetospheric magnetic field. In other regions on the magnetopause, magnetic shear $r$ between magnetic field vectors changes the pitch-angle distribution of the penetrated particles. If magnetopause magnetic shear is large particle velocity along magnetospheric magnetic field will decrease while the transverse component will increase. Corresponding enhancement of the particle gyration radius can produce the additional particle escape from the magnetosphere.

Two regions on the magnetopause where northward IMF is parallel to the magnetospheric field can be considered as possible windows for energetic particle access to the magnetosphere. The first one is on the equatorial magnetopause, especially on its dayside part (see, e.g., Vlasova et al., 2011; Pavlov, 2013) where magnetic field is stronger than in the distant tail and captures SEPs more effectively. The second one is highlatitude region where solar wind flows past the magnetosphere and IMF drapes magnetopause (see Alexeev \& Kalegaev, 1995; Alexeev et al., 2007). The resulting particle distribution fortunately detected by POES- 17 during the 20 January 2005 SEP event onset gives evidences on energetic particle access through the both regions.

Solar energetic particles that are moving in accordance with IMF direction will enter the magnetosphere in the nearequatorial region where they become quasi-trapped in much stronger magnetospheric magnetic field (see Pereyaslova, 1982; Scholer, 1975; Selesnick et al., 2014 and references therein). One can expect that more energetic particles penetrate deeper into the magnetosphere due to larger Larmor radius and should be registered first during the SEP event onset.

Particles penetrated through the near-equatorial magnetopause fill the $L$-shells around the two outer maxima (No1 and No7 lines in Table 2) of the "channeled" structure (Fig. 4a). The time profile of trapped electron fluxes $\left(E_{e}>\right.$ $30 \mathrm{keV}$ ) presented in Figure 7 shows that first and fourth maxima of the "channeled" structure measured by POES-17 (Fig. 4a) belong to the closed field lines and coincide well with the outer radiation belt maxima. These are the same solar protons that were detected onboard GOES satellites (Fig. 3). One can see that particle transport in the interplanetary space and inside the magnetosphere was very fast. The ACE and GOES data show almost simultaneous sharp flux increases. POES-17 also demonstrates corresponding enhancement of the particle fluxes.

The observed energetic particle entry to the low latitudes can be explained by direct penetration and diffusive transport of fluxes deep into the inner magnetosphere. The particle access area is the near equatorial region on the dayside magnetopause where IMF and magnetospheric field are parallel. Both mechanisms exist simultaneously, however direct penetration is dominant process during onset when particle fluxes are sufficiently anisotropic. Apparently, this process is responsible for the outer maxima formation during SEP event onset. On the other hand, diffusional processes provide uniform fluxes over polar regions and we can detect them in the minima of the fluxes during one POES-17 crossing of the polar cap. More detailed study of the relationship between diffusive and direct 


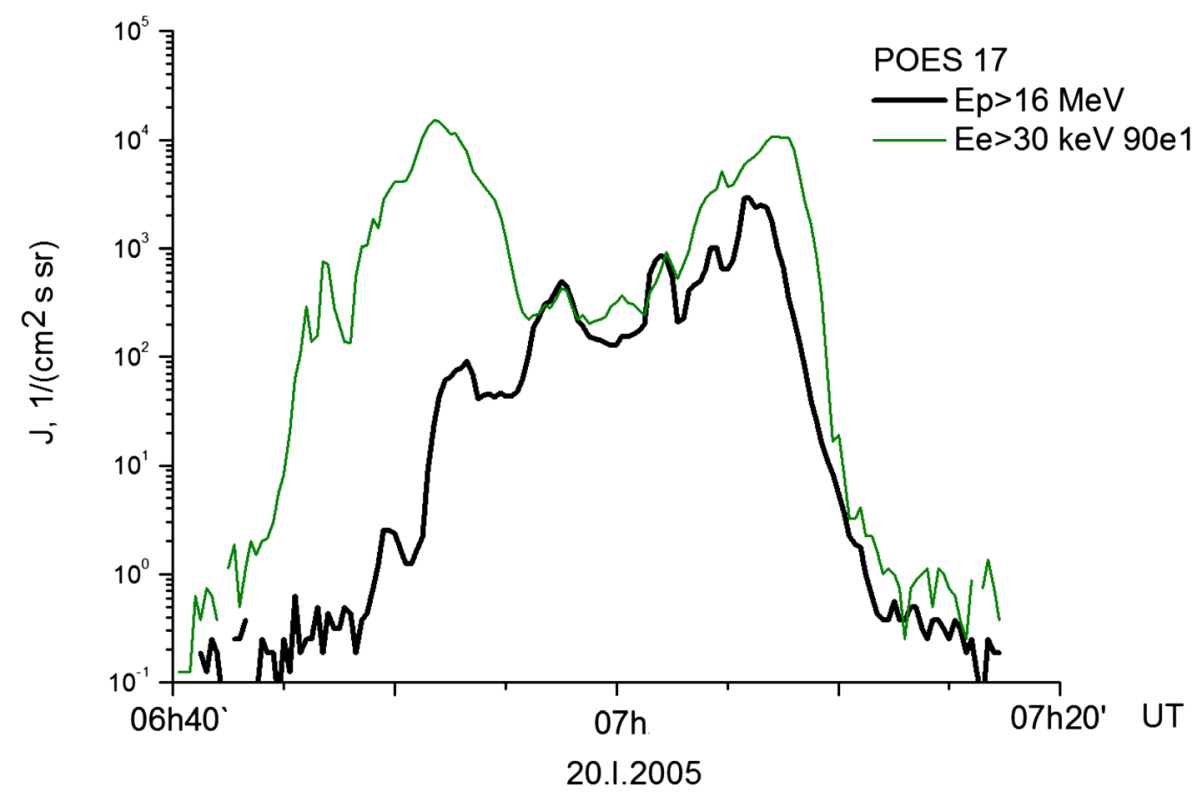

Fig. 7. Time profiles of the solar proton flux $\left(E_{p}>16 \mathrm{MeV}\right)$ and the trapped electron flux $\left(E_{e}>30 \mathrm{keV}\right)$ measured onboard POES-17.

particle penetration can be carried out based on phase space density analysis.

Two inner SEP maxima (points No3 and No5) are measured at latitudes higher than that for outer radiation belts. High energy particles directly penetrated through the high-latitude magnetopause were IMF is parallel to magnetospheric magnetic field can contribute to these fluxes. One can assume that these particles penetrate into the magnetosphere through the open field lines window at magnetopause and move along the open field lines to the opposite polar cap. One can estimate the time delay between $100 \mathrm{MeV}$ particle penetration through the magnetopause and its entry to the Earth's atmosphere (about of $30 \mathrm{~s}$ ). Particles intersect geotail current sheet where magnetic field line curvature radius is very small. In this region, particles with relatively large Larmor radii are subject of significant pitch-angle scattering. Such particles appear in the magnetosphere after penetration through the magnetopause with magnetic shear. These energetic particles, that have large gyroradii, will have opportunities for multiple pitch angle scatterings so that they become quasi-trapped.

Two "inner" high-latitude maxima are located at the same $L$-shell $(L \sim 14)$ and apparently belong to the open field lines reconnected with IMF. Figure 8 represents northern electric convection patterns reconstructed by SuperDARN measurements at UT 07:00 on January 20, 2005 (http://vt.superdarn. org/tiki-index.php?page = ASCIIData) (Greenwald et al., 2008). Polar diagram is given in the magnetic coordinates (magnetic latitude and magnetic longitude). One can see twovortex structure of electric convection in the polar cap that is usually detected under northward IMF (Burke et al., 1979; Potemra et al., 1984). Blue and red vortices correspond to regions with negative and positive electric potential in the ionosphere, respectively. They consist of closed ionospheric plasma convection patterns connected by open magnetic field lines with the southern magnetopause. Electric field of solar wind penetrates along open field lines into the magnetosphere and produces the observed distribution of the electric potential at the ionospheric level. Each of the closed convection lines has fixed electric potential, with maximum and minimum values reached at the centers of red and blue convection vortices, respectively. It is important to note that outer equipotentials of both vortices connected by magnetic field lines with high latitude magnetopause while inner ones are mapped to the low latitude magnetopause (Belenkaya, 2004; Alexeev et al., 2007).

Solid red lines show the MLT directions at 07:00 UT, so electric potential distribution presented in Figure 8 corresponds to dawn-dusk electric field in the polar cap. Ionospheric footprints of POES 17 trajectory (blue and red crosses represent minima and maxima of the fluxes measured along the orbit that is started in the evening sector and ended near noon) intersect the polar cap convectional vortex in the evening ionosphere. High-latitudinal fluxes of SEP measured by POES 17 near maxima 3 and 5 are located at $L \sim 14$ at open magnetic field lines that connected with southern high latitude magnetopause. Point 6 in the inner part of the trajectory between maxima 3 and 5 is located inside the convection vortex and apparently connected with magnetopause at middle latitudes where magnetic shear is high and SEP access is weakened.

The "open window" at the southern magnetopause is connected with polar cap in the northern hemisphere by magnetic field lines (Alexeev et al., 1993). Stable magnetospheric configuration during SEP event allows to reconstruct the pathways of penetration of solar energetic particles into the magnetosphere. For strongly northward IMF the more effective reconnection takes place near the plane $Y=0$. This region is connected by open field lines with the outer part of the ionospheric connection pattern in the evening. Here we can see also the inner maxima of SEP fluxes measured during event onset.

One can see, that potential pattern shown in Figure 8 is based on only 3 velocity vectors observed at the edge of the SuperDARN field of view and hardly represents the exact electric potential distribution. However, patterns shown in Figure 8 give information on typical potential distribution in the polar 


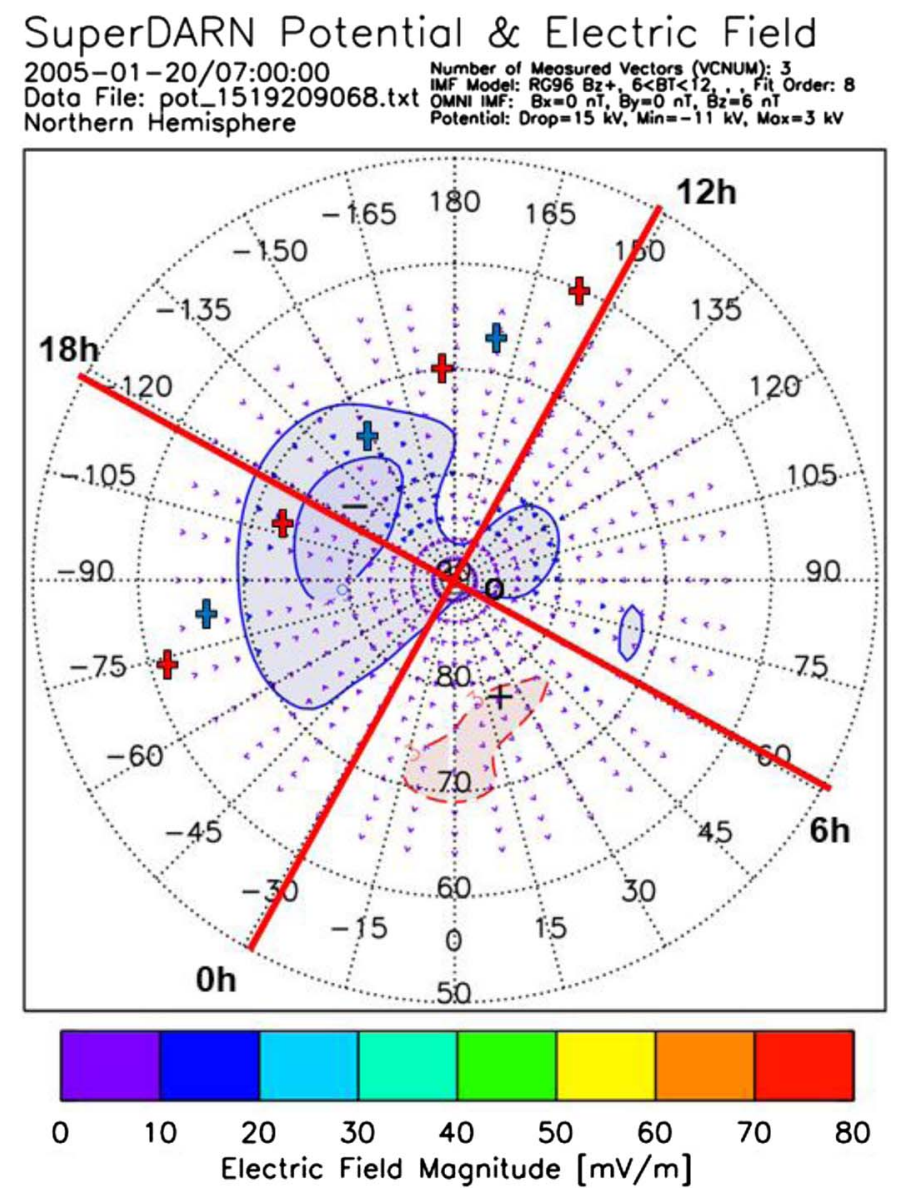

Fig. 8. Electric potential distribution in the northern polar cap at 07 UT on January 20, 2005. POES-17 is moving from the evening to the noon, trajectory is marked by crosses.

cap. The convection vortices reflect the true topology of magnetic field that helps us to better understand solar particle penetration inside the magnetosphere. In spite of some inconsistency between POES-17 data and SuperDARN diagram the last one gives the opportunity to explain of the measured particle distribution at high latitudes.

\section{Conclusion}

Dynamics features of solar energetic proton $\left(E_{p}>16 \mathrm{MeV}\right)$ penetration into the high-latitude regions of the Earth's magnetosphere were studied for January 20, 2005 solar proton event, which occurred under northward IMF condition. Complex analysis of experimental data of solar particle fluxes obtained onboard low-altitude (POES) and geosynchronous (GOES) spacecrafts and magnetic field structure calculated by A2000 quasi-dynamic magnetospheric model was carried out taking into account solar wind and interplanetary magnetic field parameters measured by ACE spacecraft.

Time profiles of solar particle fluxes measured by POES-17 spacecraft during the SEP event at UT 06:55 on January 20, 2005 had the unusual "channeled" structure. The "fresh" solar particles were located near two specific L-shells, one in the region of trapped radiation $(L \sim 4.5)$ and the other one at open field lines $(L \sim 14)$. Consequent enhancement of fluxes in four maxima registered during POES-17 polar fly-by on 6:40-7:20 UT reflects exactly the SEP event development measured by GOES satellites that confirms the same origin of particles measured by GOES and "fresh" particles measured POES-17. Simultaneously, energetic particle fluxes originated from the previous solar flare are detected at intermediate $L$-shells. The energetic spectra in these regions correspond well with the spectra measured during the previous polar cap crossing by POES-17 satellite. In contrast, energetic spectra of "fresh" particle fluxes in the vicinity of maxima of particle fluxes of the "channeled" structure are "flat". We suppose that such spectrum indicates the absence of the particles with energies below $\sim 100 \mathrm{MeV}$ in the polar magnetosphere and allows separating "old" and "fresh" particle populations during SEP event onset.

Penetration of solar particles into the Earth's magnetosphere took place under northward IMF condition at separate bundles of magnetic field lines, closed and open. Calculations of the magnetic field structure at 07:00 UT and comparison with SuperDARN observations allow to determine part of the POES-17 trajectory in high-latitude region connected with solar wind by open field lines. Calculations in terms of A2000 model of the Earth's magnetosphere give possibility to localize the regions on the near-equatorial day-side magnetopause where SEP penetration can occur. 
Acknowledgements. This study was supported by Russian Foundation of Basic Research Grant No 16-05-00760 (analysis of magnetic field structure and dynamics, N.V. and I.N.) and Russian Scientific Foundation Grant No 16-17-00098 (data analysis and effects of SEP access to magnetosphere, V.K.). The authors thank data holders of ACE, NOAA/POES and NOAA/GOES as well as World Data Center, Kyoto, for geomagnetic indices data. The authors thank SuperDARN project for data on ionospheric convection. The authors thank the Referees and the Editor very much for careful examination of our work and meaningful suggestions, which helped us to substantially improve our paper

The editor thanks Daniel Heynderickx and Shawn Young for their assistance in evaluating this paper.

\section{References}

Alexeev II, Belenkaya ES, Kalegaev VV, Lyutov Yu G. 1993. Electric fields and field - aligned current generation in the magnetosphere. J Geophys Res 98(3): 4041-4051.

Alexeev II, Kalegaev VV. 1995. Magnetic field and the plasma flow structure near the magnetopause. J Geophys Res 100: 19267-19276.

Alexeev II, Kalegaev VV, Belenkaya ES, Bobrovnikov SY, Feldstein YI, Gromova LI. 2001. Dynamic model of the magnetosphere: Case study for January 9-12, 1997. J Geophys Res 106(A11): 25683-25694.

Alexeev II, Belenkaya ES, Bobrovnikov SY, Kalegaev VV, Cumnock JA, Blomberg LG. 2007. Magnetopause mapping to the ionosphere for northward IMF. Ann Geophys 25(12): 2615-2625.

Belenkaya ES. 2004. Magnetospheric magnetic field structure under different orientation of solar wind magnetic field. Geomagn Aeron 44(4): 435-441.

Blake JB, McNab MC, Mazur JE. 2001. Solar proton polar cap intensity structures as a test of magnetic field models. Adv Space Res 28(12): 1753-1757.

Burke WJ, Kelley MC, Sagalyn RC, Smiddy M, Lai ST. 1979. Polar cap electric field structures with a northward interplanetary magnetic field. Geophys Res Lett 6(1): 21-24.

Bütikofer R, Flückiger EO, Desorgher L, Moser MR. 2008. The extreme solar cosmic ray particle event on 20 January 2005 and its influence on the radiation dose rate at aircraft altitude. Sci Total Environ 391(2): 177-183. DOI: 10.1016/j.scitotenv.2007.10.021.

Cowley SWH. 1973. A qualitative study of the reconnection between the Earth's magnetic field and an interplanetary field of arbitrary orientation. Radio Sci 8(11): 903-913.

Cowley SWH. 1983. Interpretation of observed relations between solar-wind characteristics and effects at ionospheric altitudes, in: Hultqvist B, Hagfors T, Editors. High Latitude space plasma physics, Plenum Press, New York, 225-249.

Darchieva LA, Ivanova TA, Kovrigina LM, Sosnovets EN, Tverskaya LV. 1983. Diagnosis of magnetosphere state by solar cosmic ray data. Geomagn Aeron 23(1): 62-67.

Desorgher L, Kudela K, Flückiger E, Bütikofer R, Storini M, Kalegaev V. 2009. Comparison of earth's magnetospheric magnetic field models in the context of cosmic ray physics. Acta Geophys 57(1): 75-87.

Dungey JW. 1961. Interplanetary magnetic field and the auroral zones. Phys Rev Lett 6: 47-48.

Farrugia CJ, Richardson IG, Burlaga LF, Lepping RP, Osherovich VA. 1993. Simultaneous observations of solar MeV particles in a magnetic cloud and in the Earth's northern tail lobe: Implications for the global field line topology of magnetic clouds and for the entry of solar particles into the magnetosphere during cloud passage. J Geophys Res 98(9): 15497-15507.

Flückiger EO, Smart DF, Shea MA. 1990. Determining the strength of the ring current and the magnetopause currents during the initial phase of a geomagnetic storm using cosmic ray data. $J$ Geophys Res 95(A2): 1113-1118. DOI: 10.1029/JA095iA02p01113.

Hudson MK, Kress BT, Mazur JE, Perry KL, Slocum PL. 2004. 3D modeling of shock-induced trapping of solar energetic particles in the Earth's magnetosphere. J Atmos Sol Terr Phys 66: 1389-1397. DOI: $10.1016 /$ j.jastp.2004.03.024.

Greenwald RA, Oksavik K, Barnes R, Ruohoniemi JM, Baker J, Talaat ER. 2008. First radar measurements of ionospheric electric fields at sub-second temporal resolution. Geophys Res Lett 35, L03111. DOI: 10.1029/2007GL032164.

Harnett EM. 2010. Deflection and enhancement of solar energy particle flux at the Moon by structures within the terrestrial magnetosphere. J Geophys Res 115: A01210. DOI: 10.1029/ 2009JA014209.

Kress BT, Hudson MK, Slocum PL. 2005. Impulsive solar energetic ion trapping in the magnetosphere during geomagnetic storms. Geophys Res Lett 32(6): L06108. DOI: 10.1029/2005GL022373.

Kudela K, Bučik R, Bobík P. 2008. On transmissivity of low energy cosmic rays in disturbed magnetosphere. Adv Space Res 42(7): 1300-1306. DOI: 10.1016/j.asr.2007.09.033.

Lemaire JF, Batteux SG, Slypen IN. 2005. The influence of a southward and northward turning of the interplanetary magnetic field on the geomagnetic cut-off of cosmic rays, on the mirror points positions of geomagnetically trapped particles, and on their rate of precipitations in the atmosphere. J Atmos Sol Terr Phys 67: 719-727.

Logachev YI, Bazilevskaya GA, Vashenyuk EV, Daibog EI, Ishkov $\mathrm{VN}$, et al. 2016. Catalog of solar proton events in the 23rd cycle of solar activity (1996 - 2008). Geophysical Center RAS, Moscow, Russia. DOI: 10.2205/ESDB-SAD-001, http:/www.wdcb.ru/ stp/data/SPE/katalog_SPS_23_cikla_SA.pdf

Merkin VG, Goodrich CC. 2007 . Does the polar cap area saturate? Geophys Res Lett 34: L09107. DOI: 10.1029/2007GL029357.

Miroshnichenko LI, Petrov VM. 1985. Radiation condition dynamics in space, Energoatomizdat, Moscow, 152 p.

Michel FC, Dessler AJ. 1970. Diffusive entry of solar-flare particles into geomagnetic tail. J Geophys Res 75: 6061-6072.

Morfill G, Quenby JJ. 1971. The entry of solar protons over the polar caps. Planet Space Science 19(11): 1541-1577.

Paulikas GA, Blake JB. 1969. Penetration of solar protons to synchronous altitude. $J$ Geophys Res 74: 2161-2168. DOI: 10.1029/JA074i009p02161.

Paulikas GA. 1974. Tracing of high-latitude magnetic field lines by solar particles. Rev Geophys 12(1): 117-128.

Pavlov NN. 2013. Solar energetic particles in the Earth magnetosphere: kinematic modeling of the "non-shock" penetration. J Phys Conf Ser 409: 012245. DOI: 10.1088/1742-6596/409/1/012245.

Pavlov NN, Tverskaya LV, Tverskoy BA, Chuchkov EA. 1993. Variations in the radiation belt particle flux during a strong magnetic storm of March 24, 1991. Geomagn Aeron 33(6): 41-46.

Pereyaslova NK. 1982. Solar protons in the Earth's magnetosphere, in: Energetic particles in the Earth's magnetosphere, Apatity, 3-25.

Pereyaslova NK, Nazarova MN, Petrenko IE. 1978. Features of proton spatial distribution in polar areas during solar cosmic ray events of the 21st cycle. Phys Solariterr Potsdam, (8): 107-112.

Potemra TA, Zanetti LJ, Bythrow PF, Lui ATY. 1984. By-dependent convection patterns during northward interplanetary magnetic field. J Geophys Res 89(A11): 9753-9760. 
Richard RL, El-Alaoui M, Ashour-Abdalla M, Walker RJ. 2002. Interplanetary magnetic field control of the entry of solar energetic particles into the magnetosphere. J Geophys Res 107(8): 1184. DOI: 10.1029/2001JA000099.

Richard RL, El-Alaoui M, Ashour-Abdalla M, Walker RJ. 2009. Modeling the entry and trapping of solar energetic particles in the magnetosphere during the November 24-25, 2001 storm. $J$ Geophys Res 114: A04210. DOI: 10.1029/2007JA012823.

Rodriguez JV. 2012. Undulations in MeV solar energetic particle fluxes in Earth's magnetosphere associated with substorm magnetic field reconfigurations. $J$ Geophys Res 117: A06229. DOI: 10.1029/2012JA017618.

Shue J-H, Chao JK, Fu HC, Khurana KK, Russell CT, Singer HJ, Song P. 1998. Magnetopause location under extreme solar wind conditions. J Geophys Res 103: 17691-17700.

Scholer M. 1975. Transport of energetic solar particles on closed magnetospheric field lines. Space Sci Rev 17(1): 3-44.

Selesnick RS, Baker DN, Jaynes AN, Li X, Kanekal SG, Hudson MK, Kress BT. 2014. Observations of the inner radiation belt: CRAND and trapped solar protons. J Geophys Res (Space Phys) 119(8): 6541-6552.
Störmer C. 1955. The polar aurora. University Press, Oxford.

Tsyganenko NA. 2002. A model of the near magnetosphere with a dawn-dusk asymmetry: 2. Parameterization and fitting to observations. J Geophys Res 107: A03208. DOI: 10.1029/ 2001JA000220.

Tsyganenko NA, Sitnov MI. 2005. Modeling the dynamics of the inner magnetosphere during strong geomagnetic storms. J Geophys Res 110: DOI: 10.1029/2004JA010798.

Tverskaya LV. 2011. Diagnosis of the magnetosphere by relativistic electrons of the outer belt and by solar proton penetration. Geomagn Aeron 51(1): 8-24.

Tverskoy BA. 1969. Basic mechanisms of Earth's radiation belt formation. Rev Gephys 7(1-2): 219-231.

Tverskoy BA, Darchieva LA, Ivanova TA, Kovalskaya IY, Panasyuk MI, Sosnovets EN, Tverskaya LV, Teltsov MV. 1973. Solar cosmic ray penetration into the Earth's magnetosphere. V Leningrad International Seminar, Leningrad, 367-385.

Vlasova NA, Pavlov NN, Panasyuk MI, Vedenkin NN, Ivanova TA, Lyubimov GP, Reizman SY, Tulupov VI. 2011. Some features of solar cosmic ray penetration into the earth's magnetosphere. Cosm Res 49(6): 485-499.

Cite this article as: Kalegaev VV, Vlasova NA, Nazarkov IS \& Melkova SA 2018. Magnetospheric access for solar protons during the January 2005 SEP event. J. Space Weather Space Clim. 8, A55. 